\title{
Análise de sistemas de serviços de saúde: um estudo comparativo
}

\author{
Diego Augusto Vieira Rodrigues, Gustavo Alencar Bisinotto
}

\begin{abstract}
Resumo - O atual sistema de saúde brasileiro é resultado de um processo de evolução ao longo dos anos que, em especial, nas últimas décadas, contou com investimentos substanciais para a melhoria dos serviços prestados, contudo, diversos problemas, como demora em marcações de procedimentos e superlotação, ainda são observados. Nesse contexto, considerando-se os sistemas de saúde como sistemas a eventos discretos, pode-se explorar técnicas de modelagem e de análise desenvolvidas para esta classe de sistemas. Assim, neste trabalho, são consideradas duas abordagens distintas, para modelagem e análise de sistemas de saúde, uma baseada em Rede de Petri e outra em simulação discreta com pacotes (softwares) de simulação.
\end{abstract}

Palavras-chave - Sistemas de serviços de saúde; Modelagem; Análise de sistemas; Redes de Petri interpretadas; Simulação discreta

\section{Introdução}

A organização do sistema de saúde brasileiro sofreu grandes modificações ao longo de sua evolução, de tal forma, que, atualmente, baseia-se numa rede de estabelecimentos públicos e em conjunto com estabelecimentos privados complementares, constituindo o Sistema Único de Saúde (SUS) (GOUVEIA, 2009). No intuito de buscar um constante aprimoramento dos serviços oferecidos, os investimentos públicos no setor apresentaram um crescimento acentuado no decorrer dos anos da última década, entretanto, problemas como: demora em marcações de consultas, exames, cirurgias e outros procedimentos, filas longas, falta de profissionais, superlotação, ainda são questões não resolvidas e que são enfrentadas diariamente pelos usuários (ARCANJO; AMARAL, 2015).

Nesse sentido, a gestão hospitalar dos recursos relacionados aos serviços de saúde se torna um fator de destaque à medida que a necessidade de melhoria da gestão hospitalar se torna mais latente, buscando gerir recursos muitas vezes escassos e de altos custos com eficiência (KLEN; GUIMARÃES; PEREIRA, 2008).

Além disso, dada a característica de os processos hospitalares serem bastante dinâmicos, complexos, burocráticos e fortemente dependentes dos recursos humanos, e que tais variáveis estão intimamente interligadas, de tal forma que qualquer mudança em uma dessas pode trazer para a instituição benefícios ou perdas financeiras, humanas, técnicas e diminuição da eficiência do atendimento (KLEN; GUIMARÃES; PEREIRA, 2008) (AHLERT et al., 2009).

Assim sendo, tem-se que, nos últimos anos, no ambiente de produtivo, várias técnicas de gestão foram desenvolvidas para gerenciar problemas na produção (p. ex.: Teoria de filas e processo de Markov). E que tais metodologias, eficientes na gerência de processos, encontram como desafio se adequar a novas necessidades emergentes na gestão de serviços, como na área de saúde (GONÇALVES et al., 2005). Entretanto, em comparação com serviços de saúde, um processo pode ser mais facilmente padronizado, dado que uma gama infinita de variáveis, intrínsecas à sistemas de saúde, podem se combinar de tantas formas distintas que se torna impossível prever todas as possibilidades (AHLERT et al., 2009). Dessa forma, verifica-se que a transição das técnicas não é imediata, justificando um estudo específico para a área da saúde.

Para tanto, dada a frequente e complexa variabilidade com influência de natureza aleatória no comportamento dos sistemas de saúde, um estudo analítico do problema apresenta sérias dificuldades (GONÇALVES et al., 2005). Aliado a isso, uma vez que os componentes de um sistema de serviços de saúde (pacientes, profissionais da saúde e recursos) interagem de forma discreta, em que 
suas atividades podem ser mapeadas por meio de estados, representando a situação do sistema em um instante, e cuja evolução pode ser definida pela ocorrência de eventos discretos, representando o seu comportamento dinâmico (MIYAGI et al., 2001) (MIYAGI; MIYAGI; KISIL, 2002), tem-se que sistemas de saúde podem ser tratados como Sistemas a Eventos Discretos (SEDs), possibilitando o uso de toda uma teoria de modelagem e análise consolidada.

É nesse contexto, que se ambienta este trabalho, no qual se pretende explorar duas metodologias distintas para modelagem e análise de sistemas, com enfoque em serviços de saúde, no sentido de identificar e avaliar as diferentes técnicas empregadas em cada uma.

Assim sendo, apresenta-se inicialmente uma revisão bibliográfica contendo definições fundamentais no contexto deste trabalho, juntamente com a teoria de Redes de Petri interpretadas, com introdução da metodologia Production Flow Schema (PFS) / Mark Flow Graph (MFG), e a teoria de simulação discreta. Em seguida, é feita aplicação das metodologias para a análise de sistemas de serviços de saúde, bem como a discussão de suas diferenças, por fim, expõem-se as principais conclusões oriundas do trabalho.

\section{Revisão bibliográfica}

\subsection{Serviços de saúde}

De acordo com o Dicionário Aurélio da Língua Portuguesa, serviço por ser definido como: “atividade econômica que não resulta produto tangível (p. ex.: transporte urbano; atividades de médicos, advogados, professores; administração pública), em contraste com a produção de mercadorias". Nesse sentido, dada a intangibilidade característica dos serviços, associada à simultaneidade entre sua produção e o consumo e a interação com o cliente, os serviços não podem ser estocados, como ocorre com mercadorias, perdendo assim, na ausência de demanda, sua capacidade de realização (SABBADINI; GONÇALVES; DE OLIVEIRA, 2006).

Aplicando esses conceitos na área da saúde, tem-se que, segundo a Organização Mundial da Saúde, "serviços de saúde abrangem todos os serviços que lidem com diagnóstico e tratamento de doenças, ou promoção, manutenção e restauração da saúde". Dessa maneira, "os serviços de saúde são as funções mais visíveis de qualquer sistema de saúde, e a prestação de serviços refere-se à maneira como insumos, dinheiro, profissionais, equipamentos e medicamentos são combinados para permitir o fornecimento de intervenções de saúde". De tal forma que, "a melhoria do acesso, da cobertura e da qualidade dos serviços depende da disponibilidade desses recursos chave, da forma como os serviços são organizados e geridos e dos incentivos que influenciam os fornecedores e usuários".

\subsection{Definições fundamentais}

Apresenta-se a seguir a definição de termos que envolvem conceitos básicos considerados no trabalho desenvolvido.

- Sistema: definido como grupo de objetos que estão integrados conforme com uma relação de interdependência para atingir certos objetivos (MIYAGI, 2006) (CASSANDRAS; LAFORTUNE, 2008). No caso desse trabalho, isto é, sistemas de serviço de saúde, o sistema pode ser entendido como o conjunto de entidades que abrange pacientes, profissionais de saúde, toda a infraestrutura e os dispositivos que possibilitem o fornecimento do serviço.

- Análise de sistemas: compreende o diagnóstico das necessidades, além da definição de todo o fluxo de atividades do sistema, entidades, atributos e estados possíveis do sistema (MIYAGI, 2007). No caso de sistemas de serviço de saúde, envolve toda a lógica que rege a prestação do serviço de saúde, bem como os recursos necessários e o levantamento de dados estatísticos a respeito de cada atendimento dos pacientes.

- Controle de sistemas: definido como a aplicação de uma ação pré-planejada ou uma lógica fixa no sentido de que o sistema apresente um comportamento desejado (MIYAGI, 2007). Para esse trabalho, diz respeito a todas as ações que realizadas para a manutenção do adequado fluxo de aten- 
dimento dos pacientes, desde registros, triagem e distribuição dos pacientes, ao atendimento propriamente dito.

- Níveis de controle: trata-se de uma estrutura hierárquica em níveis ou camadas das tarefa/atividades/funções do sistema (MIYAGI, 2007). No contexto desse trabalho, envolve a estrutura dos serviços realizados no sistema de saúde.

- Método: definido como um conjunto de atividades sistemáticas e racionais que permitem alcançar um objetivo (LAKATOS; MARCONI, 2010). Para o caso em questão, pode ser compreendido como os passos a serem seguidos, e o que deve ser realizado, e levado em conta, em cada um deles para a obtenção do modelo e posterior análise do sistema em estudo.

- Procedimento: definido como "a ordem na qual os fenômenos ocorrem" (MIYAGI, 2007). Na realidade desse trabalho, abrange a sequência de etapas que devem ser realizadas na metodologia para a modelagem e análise do sistema.

- Técnica: trata-se dos processos práticos que devem ser realizados para atingir o objetivo (MIRANDA NETO, 2005). Nesse contexto, refere-se às técnicas de modelagem e análise de sistemas que são utilizadas em casa uma das metodologias analisadas.

- Ferramenta: compreende recursos materiais (como: equipamentos, máquinas, infraestrutura, softwares, etc.) indispensáveis para a satisfação de tarefas inerentes ao sistema (MIYAGI, 2007). Para esse trabalho, envolve também os formulários, sistemas de cadastro, todo o maquinário e instrumental médico, entre outros.

\subsection{Redes de Petri Interpretadas}

A primeira técnica explorada nesse trabalho para a análise e modelagem de sistemas de serviços de saúde é a interpretação de redes de Petri (RdPs), que, por ser baseada em poucos elementos estruturais, possuir uma representação gráfica que possibilita o diálogo entre equipes multidisciplinares, permitir a construção de modelos em diferentes níveis hierárquicos de abstração, além de poder representar situações como: conflito, paralelismos, sequências, entre outras, mostra-se como uma alternativa para modelagem e análise do serviço de saúde (MIYAGI; MIYAGI; KISIL, 2002).

Figura 1 Elementos estruturais da RdP

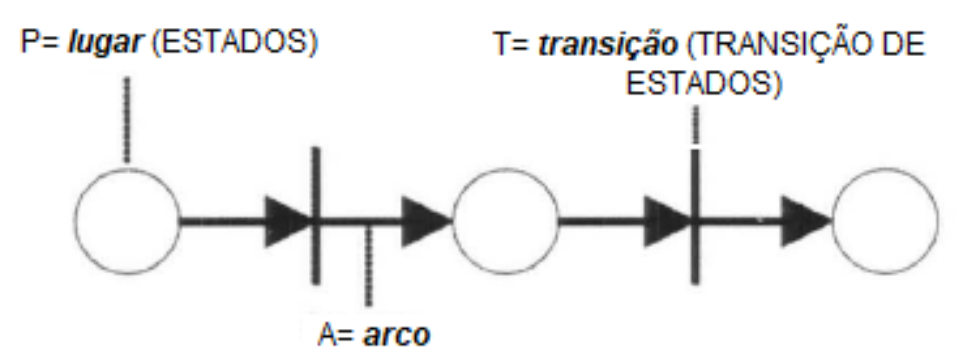

Fonte: MIYAGI, MIYAGI, KISIL (2002)

Contudo, percebe-se que uma grande quantidade de fatores deve ser levada em conta nas RdPs, tais como a quantidade (e quais são) de elementos envolvidos, o comportamento desses em cada situação, a situação inicial, entre outras, de tal forma que a "síntese em passo único" de RdPs possa ser complicada e levar a erros em sistemas de maior complexidade. Assim sendo, pode-se considerar para uma abordagem inicial de modelagem a utilização de interpretações dessas redes, e a partir de então desenvolver um detalhamento gradativo (MIYAGI, 2007).

Dessa forma, apresentam-se as técnicas do PFS (Production Flow Schema) e MFG (Mark Flow Graph), que, tratam-se de versões das RdPs para a utilização em diferentes níveis de modelagem e análise de SEDs (MIYAGI, 2007).

\section{A PFS}

É uma interpretação para a representação num nível de abstração do sistema considerando um mo- 
delo estático, isto é, sem considerar a dinâmica, assim, existe um adiamento no comprometimento do modelo, evitando uma restrição indesejável para uma primeira modelagem (MIYAGI; MIYAGI; KISIL, 2002).

A definição do PFS é feita com base nos seguintes elementos estruturais (MIYAGI, 2007):

- Atividade: componente ativo responsável pela produção, transporte e modificação de itens, representado graficamente por dois colchetes;

- Distribuidor: componente passivo capaz de armazenar, permanecer em certos estados e tornar visíveis os itens, representados por círculos;

- Arco orientado: indicam uma conexão lógica, proximidade física, direito de acesso e conexões diretas, nunca representa um componente real do sistema, mas sim uma relação lógica abstrata, representado por uma seta.

\section{B $\quad M F G$}

É uma interpretação onde se detalha o funcionamento dos processos ou serviços no sistema, isto é, o comportamento dinâmico do sistema é representado (MIYAGI; MIYAGI; KISIL, 2002).

A representação do MFG é feita com base nos seguintes elementos (MIYAGI, 2007):

- Box: indica uma condição, representado graficamente por um bloco quadrado;

- Transição: indica um evento, representado por uma barra vertical;

- Arco orientado: conecta boxes e transições em uma condição e os pré e pós-eventos que o definem, representado por uma seta;

- Porta (gate): indica a habilitação ou inibição da ocorrência de eventos correspondentes às transições, representada por um círculo negro na extremidade conectada à transição (porta inibidora) ou por um círculo branco na extremidade conectada à transição (porta habilitadora);

- Marca: indica a manutenção de uma condição, representada por um ponto negro no interior do box;

- Arco de sinal de saída: indica o envio de um sinal binário do box para um dispositivo externo, representado por uma linha que conecta os dois elementos.

Por ser derivado de Redes de Petri do tipo Condição/Evento, o MFG "herda" a capacidade de modelagem e análise da RdP (MIYAGI, 2007).

\subsection{Simulação discreta}

Em seguida, será explorado o uso da simulação discreta para o estudo do sistema, assim, tem-se que simulação pode ser compreendida como uma "imitação" de uma operação do mundo real ou sistema no tempo, envolvendo a geração de um histórico artificial do sistema, a partir da qual são inferidas as características operacionais do sistema real (BANKS et al., 2004) (MIYAGI, 2006).

Assim sendo, como na simulação ocorre uma "imitação" da realidade, pode-se, com maior ou menor sofisticação, abstrair com menor custo ou menos recursos o que acontece no sistema real, para tanto, as saídas de uma simulação devem corresponder diretamente às saídas que seriam obtidas no sistema real (BANKS et al., 2004) (MIYAGI, 2006). Para tanto, visando reduzir custos e recursos, pode-se utilizar de pacotes (softwares) de simulação disponíveis no mercado, tais como Arena, ProModel / MedModel, entre outros.

Em especial, nesse trabalho, será dado destaque à simulação discreta, que é adequada para sistemas nos quais os estados das variáveis mudam apenas da ocorrência de eventos instantâneos, em pontos discretos do tempo (BANKS et al., 2004) (MIYAGI, 2006). 
Figura 2 Passos para um estudo por simulação

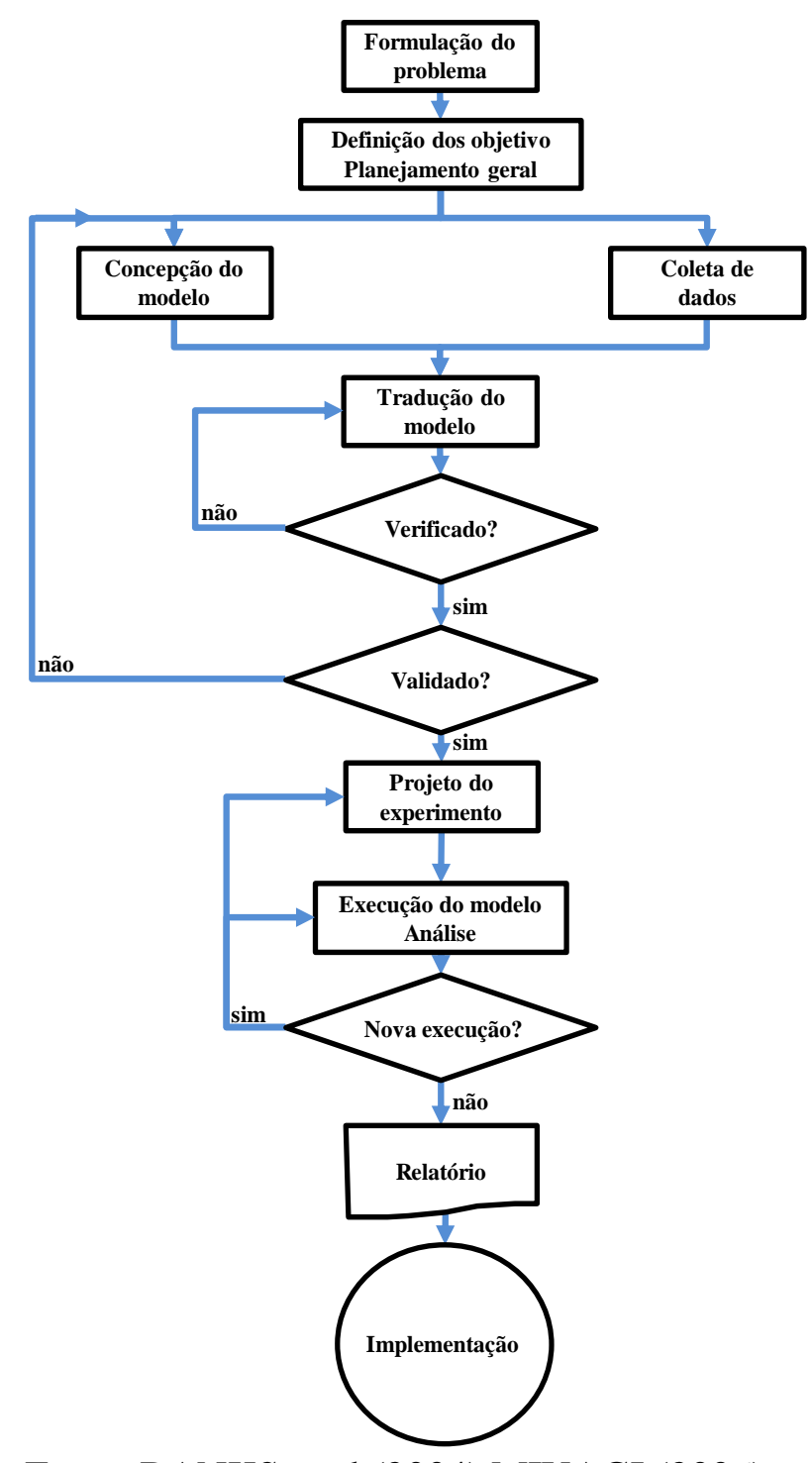

Fonte: BANKS et al (2004) MIYAGI (2006)

Dessa forma, tem-se ilustrado na figura 2 os passos para o estudo de sistemas por simulação discreta, sendo esses (BANKS et al., 2004) (MIYAGI, 2006):

- Formulação do problema: levantamento das necessidades do sistema, baseando-se em sua funcionalidade;

- Definição dos objetivos e planejamento geral: consiste nas questões que devem ser respondidas pela simulação, além de uma especificação dos meios para alcançar os objetivos, explicitando os recursos necessários;

- Concepção do modelo: construção de um modelo simples que se torna mais complexo no decorrer do projeto. Processo que deve selecionar as suposições que caracterizam o sistema e destacar os resultados de interesse;

- Coleta de dados: tomada de dados estatísticos para alimentar o modelo;

- Tradução do modelo: uso de uma ferramenta computacional adequada para a implementação do modelo;

- Verificado: comparação dos dados de saída do modelo com dados confiáveis do sistema real para comprovar a coerência do modelo;

- Validado: confirmação de que um modelo é uma representação adequada do sistema real. Esta pode ser realizada iterativamente para refinamento dos resultados até que se obtenha um resultado aceitável; 
- Projeto do experimento: detalhamento dos cenários a serem simulados, que devem ser função de um histórico de cenários previamente obtido e analisado;

- Execução do modelo e análise: a execução e análise do modelo são utilizadas para estimar medidas de desempenho para o sistema que está sendo simulado;

- Nova execução: verificação das necessidades de novas execuções, baseando-se na análise das execuções realizadas e na consistência das especificações levantadas.

- Relatórios: o código da simulação e os experimentos devem ser documentados para garantir sua preservação e no caso de eventuais revisões;

- Implementação: aplicação dos resultados da simulação no sistema real.

\section{Aplicação das metodologias em sistemas de serviços de saúde}

\subsection{Metodologia PFS / MFG}

A abordagem utilizando a metodologia PFS / MFG foi realizada em um estudo de caso no Hospital das Clínicas (HC) da Universidade de São Paulo, e nesse caso, buscava "identificar e analisar os fluxos de pacientes que procuram o atendimento do Ambulatório, que são os pacientes não urgentes e, particularmente, daqueles que procuram o serviço pela primeira vez, que são os pacientes do Ambulatório Breve" (MIYAGI; MIYAGI; KISIL, 2002).

Dessa forma, para modelagem, análise, simulação e avaliação do procedimento proposto para auxílio da tomada de decisão, seguiram-se os seguintes passos (MIYAGI; MIYAGI; KISIL, 2002):

Inicialmente com a obtenção de dados, informações e definições necessárias:

- Identificação dos fluxos de pacientes dentro do ambulatório, baseando-se nas informações obtidas junto aos profissionais do $\mathrm{HC}$;

- Definição dos objetivos da modelagem e da análise qualitativa do fluxo de pacientes;

- Coleta de dados do Ambulatório, que dado o caráter qualitativo do trabalho, foram colhidos dados suficientes apenas para a validação da metodologia proposta.

A partir de então, puderam-se seguir as etapas para a aplicação da metodologia PFS / MFG:

- Identificação da sequência de processos como fluxo principal;

- Listagem dos componentes do sistema (serviços, recursos, processos, locais, eventos, regras, etc.);

- Consideração de pacientes como itens para o PFS;

- Modelagem dos locais possíveis aos pacientes como elementos passivos, representando pré e pós condições dos processos no PFS;

- Elaboração do PFS explicitando atividades, interatividades e suas relações;

- Derivação do MFG correspondente a cada detalhamento necessário conforme uma abordagem hierárquica e modular.

Em seguida é realizada a verificação do modelo proposto e sua validação, a partir da qual são estudadas melhorias, nesse sentido:

- Verifica-se o modelo através de simulação, pela evolução das marcas no MFG, para tanto, utiliza-se de pacotes de simulação para o grafo do MFG disponíveis;

- Valida-se o modelo por meio da interpretação dos resultados, com o auxílio de uma equipe multidisciplinar de especialistas e a comparação com a dinâmica do sistema real;

- Pesquisam-se alternativas para o aperfeiçoamento do modelo;

- Simulam-se as alternativas e escolhe-se a melhor combinação delas.

Seguindo essa metodologia, obtiveram-se modelos em PFS / MFG gerais para representar o funcionamento do ambulatório, e outros mais específicos de cada atividade, como mostrado na figura 3: 
Figura 3 MFG da atividade [Recepção HC - atendimento]

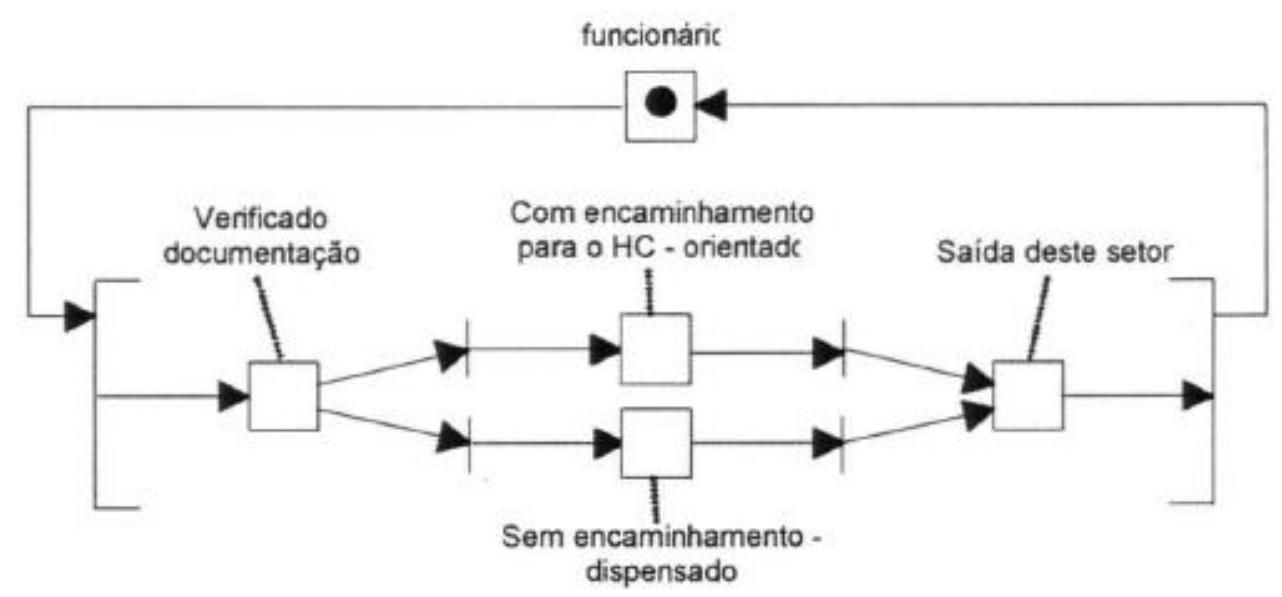

Fonte: MIYAGI, MIYAGI, KISIL (2002)

Nesse caso, pode-se observar o conflito na escolha de duas opções após o paciente ter sua documentação verificada, bem como a sincronização após o conflito, além disso, com a marcação presente no recurso "funcionário", percebe-se que essa atividade o "utiliza" para sua execução.

A partir de diversos outros modelos semelhantes a esses, e sua posterior simulação por evolução das marcas, pode-se ter uma ideia bastante clara do funcionamento do sistema, e a partir de então propor possíveis melhorias no sentido de agilizar o fluxo de pacientes, entre essas, tem-se:

- Eliminação de uma etapa de registro do paciente com verificação de documentos, elaboração de ficha e etiquetas;

- Propostas de reuniões com gerentes dos diferentes setores para discussão de divergências dos dados registrados;

- "Identificação" de pacientes e recursos para auxílio do estudo do fluxo, conforme a viabilidade econômica.

\subsection{Simulação discreta}

Já para a abordagem de simulação discreta, o estudo foi realizado no Hospital Municipal de Emergência Henrique Sérgio Gregori (HMHESG) e tem o enfoque na gestão de capacidade para a melhoria no serviço de saúde.

Como procedimento para utilização de simulação e, com isso, análise do modelo proposto, utilizou-se dos passos apresentado na figura 2. A seguir, seguem as etapas dessa metodologia (SABBADINI; GONÇALVES; DE OLIVEIRA, 2006):

\section{A Definição do problema}

O problema encontrado é o do congestionamento da unidade de emergência por uma elevada taxa de chegada de pacientes ambulatoriais causando sobrecarga sobre os serviços e a equipe médica, e o tempo de espera para receber um serviço implica diretamente na avaliação da qualidade deste e posteriormente no desempenho dos prestadores.

\section{B Estabelecimento dos objetivos}

Avaliação das medições da capacidade de atendimento, relativas a várias combinações de médicos por clínica especializada, ao longo do tempo, do setor de Pronto-Atendimento.

\section{Desenvolvimento do modelo conceitual}

O modelo conceitual foi construído a partir da análise do fluxo de pacientes no setor de prontoatendimento e de pronto-socorro da unidade de emergência do HMHESG. 
D Coleta de dados

O estudo realiza a coleta de dados estatísticos em 3 etapas:

- Elaboração e análise do fluxo de atendimento a pacientes;

- Definição da taxa de chegada dos pacientes ambulatoriais e urgentes e o tempo de atendimento;

- Coleta de dados relativos às taxas de chegada de pacientes e os tempos de atendimento em dias típicos de operação da unidade de emergência.

A partir dos dados coletados, foram identificadas distribuições de probabilidade que se ajustassem para pacientes e diferentes tipos de médicos.

Para dar maior consistência e confiabilidade ao modelo e devido à indisponibilidade de um banco de dados na HMEHSG, foi efetuado um estudo da demanda por atendimento na unidade de emergência. O universo considerado no estudo foram os 70.480 registros, compilados e analisados a partir do livro de registro de atendimentos na unidade de emergência, compreendendo o total dos pacientes relativos ao ano de 2004 .

\section{E Codificação}

O software utilizado para traduzir o modelo foi o MedModel 3.5, que permite uma representação gráfica dos componentes do sistema.

\section{F Validação do modelo}

A validação do modelo foi realizada junto à equipe médica e de gestão do hospital, que considerou o modelo como uma representação válida do sistema real.

\section{$G$ Verificação do modelo}

Para a verificação do modelo foi realizado o seguinte procedimento:

- A sequência lógica e os roteiros das entidades foram comparados com o fluxo de pacientes,

- Foi utilizada a opção de rastreamento do MedModel 3.5, o que permitiu o acompanhamento no modelo evento a evento;

- O experimento foi realizado diversas vezes. Os relatórios gerados foram comparados a situações conhecidas e analisados até que replicassem o modelo.

\section{H Experimentação}

As experiências visaram a variação de combinações de membros da equipe médica num determinado período de tempo (16h). As possíveis soluções estão na Tabela I.

Tabela I Análise de cenários

\begin{tabular}{|c|c|}
\hline Alternativa & Configuração \\
\hline 1 & 01 Clínico Geral, 01 Pediatra, 01 Cirurgião e 01 Ortopedista \\
\hline 2 & 02 Clínicos gerais, 01 Pediatra, 01 Ortopedista \\
\hline 3 & 01 Clínico geral, 02 Pediatras, 01 Ortopedista \\
\hline 4 & 02 Clínicos gerais e 02 Pediatras \\
\hline
\end{tabular}

Fonte: SABBADINI, GONÇALVES, DE OLIVEIRA (2006)

\section{Execução da simulação e análise dos resultados}

Os resultados da simulação podem ser vistos na figura 4, onde foram realizadas 100 replicações da simulação e medido o tempo médio de espera no atendimento. 
Figura 4 Tempo médio de espera

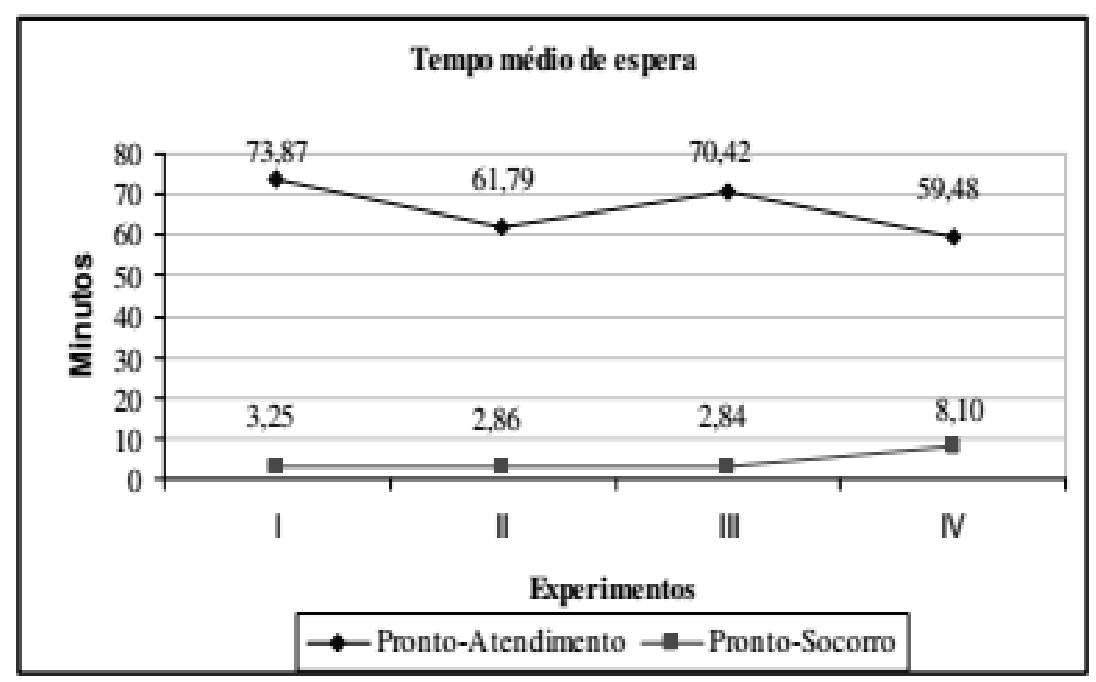

Fonte: SABBADINI, GONÇALVES, DE OLIVEIRA (2006)

\section{J Documentação, relatório de resultados e implementação}

A concepção, elaboração e desenvolvimento do modelo foram documentados e entregues aos gestores do hospital. Com base no estudo realizado, a administração do hospital alterou sua estrutura de funcionamento, adotando as soluções apresentadas no estudo.

\section{Discussão}

Apresentadas as duas metodologias para modelagem e análise do sistema de serviços de saúde, percebem-se algumas diferenças bastante evidentes entre elas, apesar de ambas terem como objetivo obtenção de modelos e verificação do comportamento dos sistemas, para uma posterior análise de melhorias.

Assim, nota-se, por exemplo, que a abordagem baseada na metodologia PFS / MFG apresenta uma vantagem em relação à simulação quando a quantidade de dados disponíveis é escassa, ou seja, o conhecimento do sistema ser mais superficial, uma vez que o modelo em PFS é hierárquico e pode ser tão detalhado quanto possível conforme o conhecimento do sistema em estudo e os dados a respeito do mesmo.

Nesse sentido, observa-se que dado o detalhamento progressivo do modelo em PFS, pode-se identificar já durante a modelagem alguma questão estrutural que venha a implicar em um aprimoramento do sistema, além de possibilitar que durante o processo de modelagem sejam detectados, e corrigidos possíveis erros, antes de ocorrer a simulação que retornaria resultados incompatíveis com a realidade.

Além disso, o fato de a simulação necessitar do uso de estatísticas torna necessário, para confiança nos resultados, um longo histórico de aferimento de dados, o que nem sempre é possível.

Contudo, no caso de haver dados suficientes e de confiança, a simulação discreta fornece alguns resultados mais concretos no sentido de melhor alocação de serviços, possibilitando um conhecimento mais exato de, por exemplo, em quais períodos ocorrem maior solicitação de certo recurso, seja profissional ou algum maquinário, ou quais recursos ficam sobrecarregados enquanto outros se encontram disponíveis.

Por fim, ambas apresentam um forte enfoque gráfico, o que facilita o diálogo entre equipes multidisciplinares, e que conforme a quantidade de dados disponíveis, e o nível de abstração ou de precisão dos resultados desejados, em ambos os casos seu uso pode ser justificável e apresentar os ganhos esperados, em termos econômicos e de tempo para a análise dos sistemas. Além de ambas terem possibilitado a proposta de melhorias para o funcionamento do sistema a partir da análise dos modelos obtidos. 


\section{Conclusão}

Dessa forma, pode-se perceber que para o mesmo objetivo, de realizar a modelagem e análise de um sistema, no caso, sistemas de serviços de saúde, podem ser utilizadas, de forma válida, diversas metodologias e empregadas diferentes técnicas para se obter os resultados desejados, conforme os requisitos do trabalho ou da análise. Isso fica visível tanto pelas duas metodologias estudadas e discutidas nesse trabalho, quanto diversas outras disponíveis na literatura que versam sobre o tema, como: Teorias de Fila, Processo de Markov, além de outros pacotes de simulação como o Arena, e diversas outras abordagens.

Assim, por meio desse trabalho, pode-se observar a aplicação de diversos dos conceitos vistos na disciplina de Sistemas a Eventos Discretos, bem como a utilização de alguns pacotes de simulação (softwares) utilizados em aula, em usos concretos de engenharia, no sentido de buscar soluções para problemas que ainda são frequentes em sistemas de serviços de saúde.

\section{REFERÊNCIAS}

AHLERT, F. C. et al. Gestão de serviços na área da saúde: simulação computacional no auxílio à tomada de decisão. ENCONTRO NACIONAL DE ENGENHARIA DE PRODUÇÃO, 29., Salvador, 2009. ENEGEP 2009 : a engenharia de produção e o desenvolvimento sustentável : integrando tecnologia e gestão = La ingeniería y la producción el desarrollo sostenible : integración de tecnología y gestión $=$ The industrial engineering and the sustentable development $:$ integrating technology and management. Salvador. : ABEPRO, 2009.

ARCANJO, C. F. D.; AMARAL, T. M. Mapeamento do fluxo de pacientes e simulação de eventos discretos no sistema público de saúde: um caso prático em uma unidade de pronto atendimento em Juazeiro - BA. In: ENCONTRO NACIONAL DE ENGENHARIA DE PRODUÇÃO, 35, Fortaleza, 2015. ENEGEP: anais. Fortaleza: [s.n.], 2015.

BANKS, J. et al. Discrete Event Systems Simulation. 4a ed.Upper Saddle River, N.J.: Pearson Prentice Hall, 2004. 528 p.

CASSANDRAS, C. G.; LAFORTUNE, S. Introduction to event discrete systems. 2a ed. New York: Springer, 2008. $780 \mathrm{p}$.

FERREIRA, A. B. H. Dicionário Aurélio da língua portuguesa. 8a ed. Curitiba: Positivo, 2010. 960 p.

GONÇALVES, A. A. et al. Modelo de simulação aplicado na gestão de serviços de saúde. In: ENCONTRO NACIONAL DE ENGENHARIA DE PRODUÇÃO, 25. Porto Alegre, 2005. Anais. Porto Alegre, ABEPRO. 2005.

GOUVEIA, G. C. Avaliação da satisfação dos usuários com o sistema de saúde brasileiro. Tese (Doutorado) - Fundação Oswaldo Cruz, Recife - PE, 2009.

KLEN, A. M.; GUIMARÃES, I. F. G.; PEREIRA, D. M. A Utilização da simulação em gestão hospitalar: aplicação de um modelo computacional em um centro de imobilizações ortopédicas. In: ENCONTRO NACIONAL DE ENGENHARIA DE PRODUÇÃO, 28, Rio de Janeiro, 2008. ENEGEP 2008. Rio de Janeiro: [s.n.], 2008.

LAKATOS, E. M.; MARCONI, M. de A. Fundamentos de Metodologia científica. 7.ed. São Paulo: Atlas, 2010. 297 p.

MIRANDA NETO, M. J. Pesquisa para o Planejamento: Métodos e Técnicas. Rio de Janeiro: FGV, 2005. 84 p. 
MIYAGI, M. M. et al. Simulação discreta e redes de Petri para análise de sistemas de saúde. In: ENCONTRO NACIONAL DE ENGENHARIA DE PRODUÇÃO, 28., Niterói, 2001. ENEGEP: anais. Niterói: UFF/TEP, 2001.

MIYAGI, M. M.; MIYAGI, P.E.; KISIL, M. Modelagem e análise de serviços de saúde baseados em redes de Petri interpretadas. Revista Produção, São Paulo, v. 11, n. 2, p.23-39, 2002.

MIYAGI, P. E. Introdução a simulação discreta. São Paulo: [s.n], 2006. 52 p.

MIYAGI, P. E. Controle programável: fundamentos do controle de sistemas a eventos discretos. 3.ed. São Paulo: Edgard Blucher, 2007.

SABBADINI, F.; GONÇALVES A. A.; DE OLIVEIRA, M. J. F. (2006) Gestão da capacidade de atendimento e simulação computacional para a melhoria na alocação de recursos e no nível de serviço em hospitais. In: SIMPÓSIO DE EXCELÊNCIA EM GESTÃO E TECNOLOGIA, 3.SEGeT. [S.1.]: [s.n.] , 2006.

WORLD HEALTH ORGANIZATION. Health Services. Disponível em:

<http://www.who.int/topics/health_services/en/> Acesso em: 20 de janeiro de 2017

\title{
Title - Analysis of Health Service Systems: A Comparative Study
}

\begin{abstract}
The current Brazilian health system is the result of a process of evolution over the years that, in particular, in recent decades, counted on substantial investments for the improvement of services provided, however, several problems, such as delays in procedures marking and overcrowding, are still observed. In this context, considering health systems as systems with discrete events, it is possible to explore modeling and analysis techniques developed for this class of systems. Therefore, in this paper, two different approaches are considered for modeling and analysis of health systems, one based on Petri Net and another on discrete simulation with packages (softwares) of simulation.
\end{abstract}

Keywords - Health service systems, Modeling, Systems analysis, Interpreted Petri nets, Discrete simulation

Diego Augusto Vieira Rodrigues, estudante de graduação do curso de Engenharia Mecatrônica da Escola Politécnica da Universidade de São Paulo. Bolsista do Programa de Educação Tutorial, PET Automação e Sistemas da Escola Politécnica.

Gustavo Alencar Bisinotto, estudante de graduação do curso de Engenharia Mecatrônica da Escola Politécnica da Universidade de São Paulo. Bolsista do Programa de Educação Tutorial, PET Automação e Sistemas da Escola Politécnica. Colaborador no Laboratório Tanque de Provas Numérico (TPN-USP). 